\title{
Toxoplasmose gestacional: perfil epidemiológico e conhecimentos das gestantes atendidas na unidade básica de saúde de um município alagoano
}

\author{
Toxoplasmosis in pregnancy: Epidemiological profile and knowledge of pregnant women \\ assisted in basic units of an Alagoan municipality
}

Dayanne Silva de Moura ${ }^{1}$, Rita de Cássia Mendes Oliveiraa ${ }^{1}$, Thiago José Matos-Rocha ${ }^{2}$

\section{Resumo}

A toxoplasmose é uma zoonose mundialmente distribuída causada por um protozoário intracelular, o Toxoplasma gondii, que é capaz de infectar o homem, aves e mamiferos. A toxoplasmose congênita ocorre quando a gestante adquire a primoinfecção durante a gravidez e transmite via transplacentária ao feto. Assim o presente trabalho teve como objetivo analisar o conhecimento das gestantes frente a este assunto. Foi realizado um estudo descritivo de abordagem quantitativa realizada em uma Unidade Básica de Saúde. A amostra da pesquisa foi do tipo não probabilístico por conveniência, contemplando 40 gestantes em acompanhamento pré-natal. Frente aos resultados analisados, verificou-se que o desconhecimento das gestantes sobre a toxoplasmose está relacionado à falta de orientações e informações dos profissionais de saúde durante as consultas de pré-natal, o que justifica os hábitos de vida e alimentares inadequados favorecendo uma possivel infecção. O papel da Enfermagem é de fundamental importância neste aspecto, um pré-natal respaldado evita agravos durante gestação e pós-parto.

Descritores: Toxoplasmose congênita, Formas de transmissão, Papel do profissional de enfermagem, Gravidez, Cuidado pré-natal

\section{Abstract}

Toxoplasmosis is a worldwide spread infection caused by

1. Graduada em Enfermagem pelo Centro Universitário CESMAC 2. Professor de Parasitologia Humana da Universidade Estadual de Ciências da Saúde de Alagoas. Coordenador da Especialização de Análises Microbiológicas e Parasitológicas, Farmácia Hospitalar e Citologia Clínica no Centro Universitário CESMAC

Trabalho realizado: Centro Universitário CESMAC

Endereço para Correspondência: Thiago José Matos Rocha. Rua Cônego Machado, 918. Bairro: Farol - 57051-160 MaceióAlagoas-Brasil. Tel.: +55 (82) 3215-5137. E-mail: thy_rocha@ hotmail.com an intracellular protozoan Toxoplasma gondii, which can infect humans, birds and mammals. Congenital toxoplasmosis occurs when the mother acquires a primary infection during pregnancy and transmitted via the placenta to the fetus. Thus, the present study was to analyze the knowledge of pregnant women against this a descriptive study of qualitative and quantitative approach carried out in Basic Health Unit was conducted. The survey sample was non probabilistic for convenience, covering 40 pregnant women in prenatal care. Faced with the results analyzed, it was found that the lack of knowledge of pregnant women about toxoplasmosis is related to lack of guidance and health professionals information during prenatal consultations, which explains the life and eating habits inadequate favoring a possible infection. The role of nursing is crucial in this respect, prenatal backed prevents diseases during pregnancy and postpartum.

Keywords: Toxoplasmosis, congenital; Nurse's rule; Pregnancy; Prenatal care

\section{Introdução}

Toxoplasma gondii é um protozoário capaz de infectar a maioria das espécies de sangue quente, incluindo o homem. A toxoplasmose afeta cerca de um terço da população mundial, mas tem prevalência variável em diferentes populações, dependendo da combinação de fatores tais como clima, hábitos higiênicos, população de gatos e hábitos de preparação e ingestão de alimentos. No Brasil, a prevalência de toxoplasmose é considerada alta ${ }^{(1)}$.

Sendo os felinos os hospedeiros definitivos e suas fezes contaminadas uma forma de contaminação, o contato com estas, tem sido referido como importante fator associado à toxoplasmose ${ }^{(2)}$.

A toxoplasmose é uma das infecções mais graves durante a gravidez devido ao abortamento e ao risco de acometimento fetal. Assim, torna-se de fundamental importância o diagnóstico precoce no pré-natal ${ }^{(3)}$. 
A gestação pode ser considerada um fator de risco para a toxoplasmose. A superior vulnerabilidade ao parasita pelas gestantes pode ser atribuída a alterações imunológicas e hormonais que ocorrem durante a gestação(4).

A transmissão via placentária ou transmissão congênita, é uma das formas de transmissão da toxoplasmose, e não por acaso, a mais preocupante, em razão da alta taxa global de transmissão materno-fetal ${ }^{(5)}$.

Como a toxoplasmose é assintomática em $90 \%$ dos casos, a triagem sorológica é importante para conhecimento do perfil sorológico e definição da presença ou não do risco de toxoplasmose congênita ${ }^{(2)}$.

De acordo com Pessanha et al, 2011(6), a sorologia de toxoplasmose no início da gestação, é indispensável para um diagnóstico eficaz, onde o mesmo define o nível de acometimento fetal, porém muitas vezes não é possível, pois muitas gestantes não iniciam seu pré-natal no primeiro trimestre.

Segundo Lopes et al, 2011(7) para detecção da toxoplasmose uma das importantes ferramentas que permite a adoção de medidas profiláticas e terapêuticas precocemente e, assim, a diminuição da taxa de transmissão vertical e/ou danos ao desenvolvimento fetal, é a triagem sorológica materna.

Para muitas gestantes os fatores de risco, bem como os sinais e sintomas da infecção não são reconhecidos, sendo essencial o papel do obstetra no diagnóstico e prevenção da infecção ${ }^{(8-9)}$.

A baixa escolaridade e a consequente falta de informação sobre os cuidados preventivos para toxoplasmose tornam as gestantes propensas ao risco aumentado de infecção e subsequente transmissão ao feto ${ }^{(10-11)}$.

Assim, considerando a importância do conhecimento das gestantes sobre toxoplasmose, como uma infecção materno-fetal, entende-se que tal compreensão sobre esta parasitose intervém de forma direta nos meios de prevenção, o trabalho teve como objetivo analisar o conhecimento de gestantes em relação à toxoplasmose, como meios de transmissão, prevenção e seus hábitos alimentares e higiênicos, através de uma entrevista em uma Unidade Básica de Saúde.

\section{Material e Método}

Foi realizado um estudo descritivo de abordagem quantitativa realizada em uma Unidade Básica de Saúde, do município de Satuba-AL, durante o período de fevereiro a março de 2016. Este trabalho foi aprovado pelo comitê de ética em pesquisa do Centro Universitário Cesmac, sob CAAE: 44464215.1.0000.0039.

A amostragem da pesquisa foi do tipo não probabilística por conveniência, contemplando 40 gestantes em acompanhamento pré-natal na Unidade de Saúde, que atendessem os critérios de inclusão e exclusão, descritos abaixo.

Os critérios de inclusão abrangeram gestantes, de qualquer idade gestacional, com consultas de pré-natal agendadas em datas pré-estabelecidas e que aceitaram participar da pesquisa.

Foram excluídas do estudo às gestantes que faltaram à consulta pré-natal na data agendada, e ainda aquelas que não aceitaram assinar o termo de Consentimento Livre e Esclarecido (TCLE).

Para a coleta de dados, foi elaborado um questionário composto por 11 perguntas. Dentre elas, questões referentes a dados sóciodemográficos e perguntas baseadas nos fatores que podem expor a gestante à infecção por T. gondii, sendo elas: já ouviram falar sobre toxoplasmose? Possui gatos em casa? Há contato com o felino? Recolhe as fezes de gatos? Como o leite é consumido? Já recebeu algum tipo de orientação sobre toxoplasmose? Já realizou teste sorológico para toxoplasmose? Já sofreu aborto? Faz ingestão de carne crua? Toma água filtrada? Conhece formas de prevenção da toxoplasmose?

As gestantes foram sensibilizadas quanto à importância do projeto, e a aplicação do questionário deu-se início após a assinatura do TCLE.

Foram garantidas as gestantes envolvidas na pesquisa todos os direitos citados no TCLE, quais sejam: que a qualquer momento a participante poderá solicitar maiores esclarecimentos sobre a pesquisa, às pessoas responsáveis pela mesma; que as informações conseguidas através da participação não permitirão a identificação das envolvidas; sigilo sobre qualquer informação que possa levar à sua identificação pessoal e familiar e desistir, a qualquer tempo, de participar da pesquisa. Todas as informações coletadas foram analisadas e apresentadas em forma de gráficos.

\section{Resultado}

Mediante o gráfico 1, verificou-se que 30 das 40 gestantes analisadas tinham menos de 25 anos, 15 delas eram menores de idade.

O nível de escolaridade dessas mulheres é considerado fator importante para conhecimento e prevenção da toxoplasmose, sendo ponto indispensável do instrumento da coleta de dados. Quando perguntadas sobre o nível de escolaridade observou-se que 22 (52\%) das gestantes tinham estudado apenas o primário, $25 \%$ tinham ensino fundamental completo, $23 \%$ ensino médio completo e nenhuma tinha ensino superior.

A toxoplasmose adquire enorme relevância quando ocorre durante a gestação pelo risco de transmissão vertical. Sendo assim, fez-se necessário saber se as pesquisadas haviam passado por gestações prévias. Das 40 mulheres entrevistadas, $47 \%$ possuíam outros filhos, 


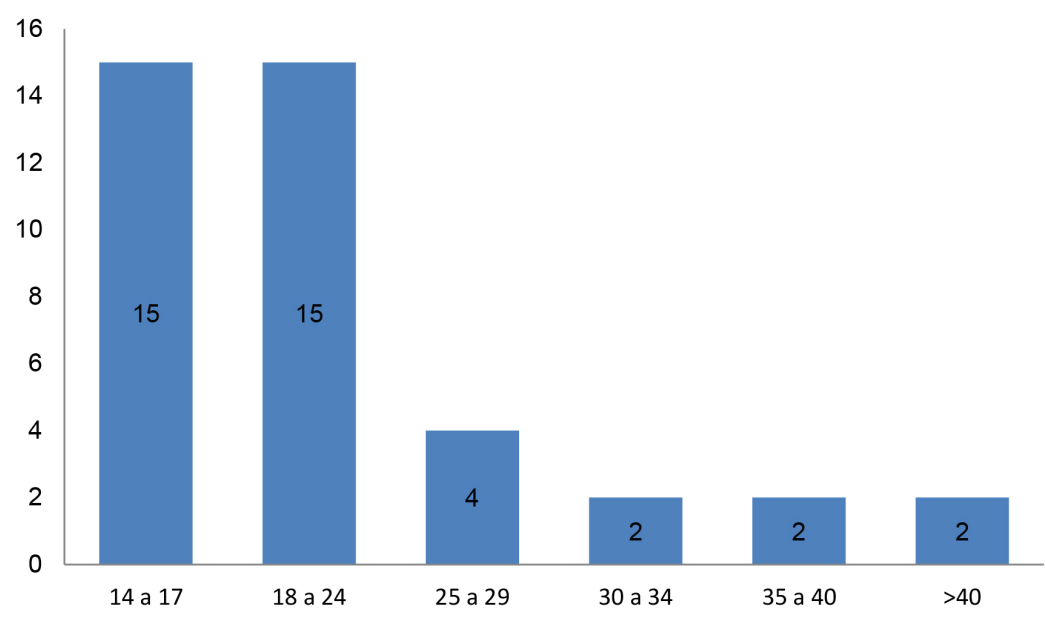

Gráfico 1 - Idade das gestantes que participaram da pesquisa atendidas em unidade básica de saúde de um município alagoano, 2016.

consequentemente tinham passado por outras gestações, enquanto $53 \%$ estavam em sua primeira gestação.

A falta de conhecimento, a respeito da toxoplasmose interfere de maneira direta nas medidas de prevenção primárias. Observou-se que 22 gestantes nunca haviam ouvido falar em toxoplasmose, apenas nove afirmaram que sim, outras nove disseram ter pouco conhecimento, gráfico 2 .

Quanto às orientações e informações as gestantes sobre esta parasitose durante as consultas de pré-natal, $85 \%$ das entrevistadas afirmaram não receber nenhum tipo de orientação ou informação sobre esta parasitose e apenas $15 \%$ afirmaram que sim. Observou-se que 22 gestantes afirmaram não ter realizado o teste sorológico para toxoplasmose, pela falta de informação e orientações das pesquisadas, no entanto, 12 relatam não lembrar ter realizado o exame e seis que sim. Quando perguntadas se já haviam sofrido algum tipo de aborto, 33 delas responderam que não, enquanto sete, afirmaram que sim.

As pesquisadas responderam que 10 delas criavam gatos, as mesmas tinham contato direto com o felino, onde oito eram responsáveis por recolher as fezes, gráfico 3.

Através da entrevista, as gestantes foram questionadas quanto à ingesta da carne, já que é um fator primordial quando se fala em toxoplasmose, $80 \%$ das entrevistadas responderam que consomem a carne cozida, bem passada e apenas $20 \%$ consomem carne crua ou malpassada. Mesmo a maioria relatando ingerir a carne bem cozida, a preocupação maior é com as que ainda consomem a carne malpassada.

Sabendo-se que a ingestão de água não filtrada pode contribuir para a infecção da toxoplasmose, perguntou-se a estas gestantes como era o consumo da água utilizada pelas mesmas. Ao serem perguntadas

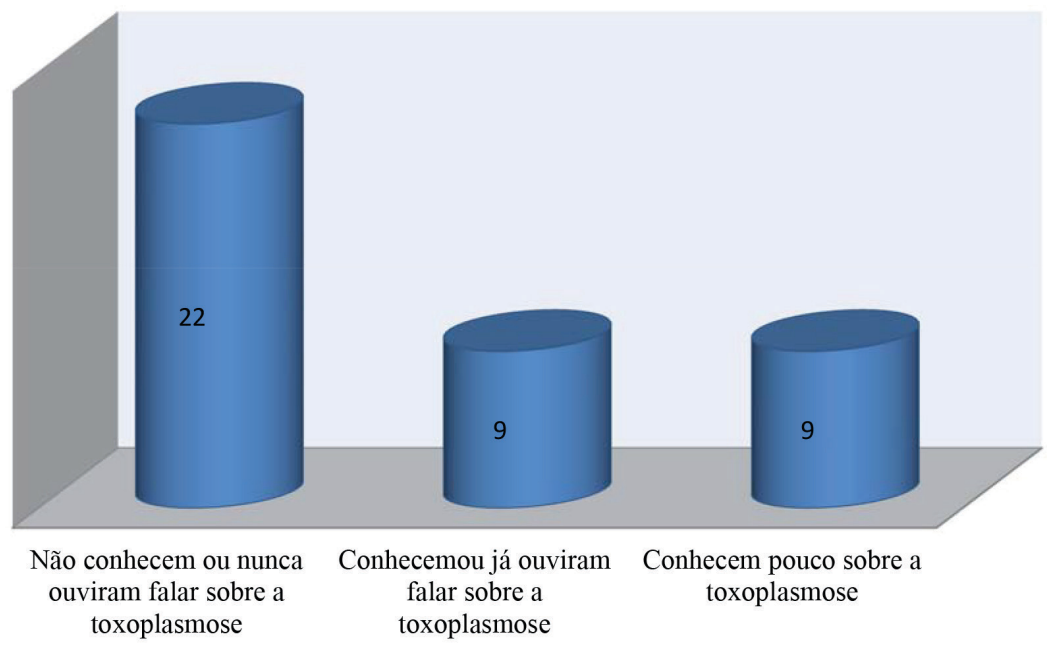

Gráfico 2 - Quantitativo de gestantes que não conhecem ou nunca ouviram falar sobre toxoplasmose, atendidas em unidade básica de saúde de um município alagoano, 2016. 


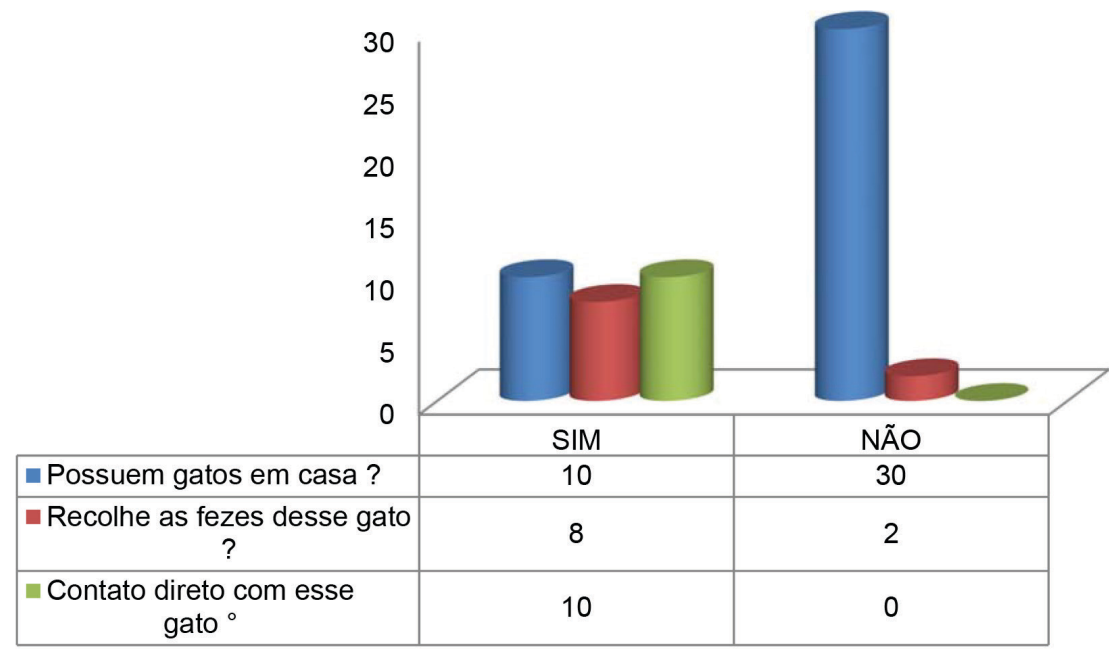

Gráfico 3 - Quantitativo de gestantes que possuem gatos em casa, e se há contato com o felino, atendidas em unidade básica de saúde de um município alagoano, 2016. Fonte: Dados da pesquisa.

se a água ingerida é filtrada ou mineral, 31 (77\%) delas responderam que sim e nove $(23 \%)$ responderam que não. Analisando as gestantes entrevistadas, notou-se que ainda existem gestantes que consomem água não filtrada. De acordo com os dados colhidos durante a pesquisa, de 40 gestantes, 16 (40\%) relataram ingerir leite fervido (gráfico 4).
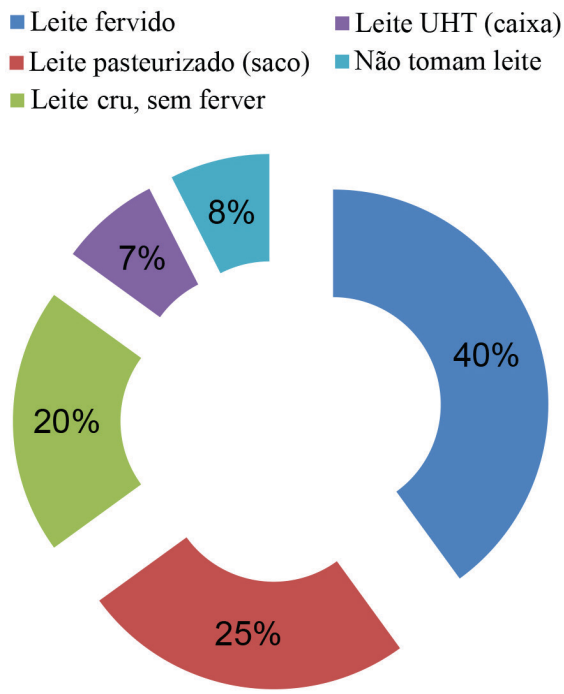

Gráfico 4 - Como é o leite consumido pelas gestantes atendidas em unidade básica de saúde de um município alagoano, 2016.

Ponto importante para a elaboração desta pesquisa é analisar o conhecimento das gestantes sobre a toxoplasmose. De acordo com os dados da pesquisa, foi observado que das 40 gestantes entrevistadas, 12 relataram que sim, conhecem algum meio de transmissão e 28 desconhecem qualquer forma de transmissão da toxoplasmose.
Procurou-se também saber qual o conhecimento das gestantes sobre meios de prevenção, já que é através deste que as gestantes irão saber como prevenir-se desta infecção. De 40 gestantes, 34 delas desconhece qualquer meio de prevenção da toxoplasmose e apenas seis conhecem formas de prevenir a toxoplasmose.

\section{Discussão}

De acordo com um estudo realizado por Inagaki et al, 2014 ${ }^{(10)}$. Observou-se que houve maior concentração de gestantes previamente infectadas pelo $T$. gondii expostas a fatores de risco conhecidos como baixo nível socioeconômico e gestação na adolescência. Sendo a idade (Gráfico 1) fator epidemiológico importante na Identificação das mulheres suscetíveis, onde o conhecimento destas possa limitar o risco de infecção durante a gravidez.

Um estudo realizado por Avelino et al, 2003 ${ }^{(12)}$ mostra que as infecções agudas com detecção de anticorpos da classe IgM ocorrem mais frequentemente em pacientes mais jovens, devendo-se dar especial atenção às gestantes adolescentes, considerando que as mesmas têm risco 7,7 vezes maior de adquirir toxoplasmose.

Resultados diferentes foram observados em um estudo feito em Rolândia e Caxias do Sul, onde gestantes de faixa etária mais elevada apresentaram maior prevalência da toxoplasmose. Acredita-se que quanto mais alta a faixa etária, maior a porcentagem de indivíduos infectados e, portanto, soropositivos. Isso pode ser atribuído à maior exposição ao parasita no decorrer dos anos ${ }^{(4)}$.

Quando perguntadas sobre o nível de escolaridade observou-se que $22(52 \%)$ das gestantes tinham estudado apenas o primário. Bittencourt et al, 2012 ${ }^{(4)}$, 
Moura DS, Oliveira RCM, Matos-Rocha TJ. Toxoplasmose gestacional: perfil epidemiológico e conhecimentos das gestantes atendidas na unidade básica de saúde de um município alagoano. Arq Med Hosp Fac Cienc Med Santa Casa São Paulo. 2018;63(2):69-76.

evidenciaram que gestantes com oito anos ou menos de escolaridade apresentaram risco 1,8 vezes mais elevado de se infectarem que as demais, evidenciando que maior grau de instrução é um fator de proteção para a infecção pelo T. gondii. Esse dado identifica a importância de investimento em educação, pois se trata de um fator importante para a prevenção da infecção e promoção da saúde.

As gestantes multíparas de Palotina apresentaram maior chance de infecção pelo $T$. gondii em relação às primigestas. $\mathrm{O}$ risco foi calculado em 1,9 vezes mais chance de adquirir a infecção ${ }^{(4)}$. Acredita-se que na população com maior escolaridade encontram-se melhores hábitos higiênicos, reduzindo a possibilidade de contaminação.

Observou-se que 22 gestantes nunca haviam ouvido falar em toxoplasmose. O desconhecimento desta parasitose é uma realidade agravante, não há como se prevenir do que não se conhece, as gestantes mostraram-se surpresas ao serem abordadas sobre o que conheciam da toxoplasmose, algumas relataram ter recebido alguma informação vaga sobre o assunto, não sabendo expor detalhes demostrando à necessidade de ações educativas ${ }^{(13)}$.

Das 40 gestantes, $72 \%$ delas, afirmaram ter realizado pré-natal em gestações anteriores, $28 \%$ delas não realizaram pré-natal. Sendo o pré-natal o momento favorável para educação em saúde, é importante que os profissionais de saúde conheçam os fatores de risco associados à aquisiç̧ão da infecção pelo T. gondii para que ofereçam informações adequadas às gestantes sobre sua prevenção ${ }^{(10)}$.

No Brasil a maioria das gestantes inicia seu pré-natal depois do primeiro trimestre de gestação, isto dificulta a detecção de uma possível infecção pelo $T$. gondii. Spalding et al ${ }^{(13)}$ consideram que da $10^{\mathrm{a}}$ à $24^{\mathrm{a}}$ semana é o período mais crítico em relação à infecção congênita. Das gestantes entrevistadas, 85\% afirmaram não receber nenhum tipo de orientação ou informação sobre esta parasitose. Um estudo realizado com as gestantes de Aracajú por Inagaki et al ${ }^{(10)}$ afirmam que aproximadamente $30 \%$ das gestantes eram suscetíveis à infecção pelo T. gondii. Isso evidencia a importância de intensificar as medidas de prevenção primária devido à possibilidade de ocorrer a soroconversão durante a gestação.

O papel do enfermeiro é de fundamental importância no acolhimento e aconselhamento as gestantes, se as mesmas não são orientadas a respeito, aumenta as chances de infecção ${ }^{(14)}$.

Um estudo realizado por Silva, Camargo Júnior, $2013^{(15)}$ afirmam que os enfermeiros e médicos possuem poucas informações sobre medidas preventivas da toxoplasmose, e os enfermeiros desconheciam informações sobre diagnostico e questões clinicas.
A orientação dessas mulheres sobre a toxoplasmose evita o risco de transmissão. É de responsabilidade do profissional de saúde atuar na orientação preventiva durante o pré-natal.

As orientações de prevenção primária devem enfatizar a importância de lavar as mãos ao manipular carne crua; evitar o consumo de carne mal cozida, água sem tratamento e leite não pasteurizado, assim como de alimentos expostos a moscas, baratas, formigas e outros insetos $^{(16)}$.

A triagem sorológica para toxoplasmose é de suma importância na gestação, pois possibilita o tratamento precoce, evitando a ocorrência de aborto, malformações congênitas e/ou problemas tardio, nesse estudo a maior parte das gestantes relatam nunca ter feito sorologia $^{(17)}$.

A prevenção à transmissão congênita da toxoplasmose consiste em acompanhar a gestante durante o pré-natal. A gestante deve submeter-se a exames laboratoriais periódicos, a fim de se detectar precocemente eventual infecção pelo T. gondii. As gestantes devem ser orientadas sobre todos os exames solicitados, bem como o porquê de cada, e os seus resultados, serem esclarecidas as gestantes, sobre as possíveis patologias e o risco para seu bebê. Um pré-natal satisfatório evita agravos e complicações para mãe e filho ${ }^{(18)}$.

Nesta pesquisa todas as entrevistadas afirmaram que não eram soropositivas para T. gondii. Mediante todos os resultados citados, é provável que o desconhecimento sobre essa parasitose as faça acreditar que nunca foram infectadas.

Quando perguntadas se já haviam sofrido algum tipo de aborto, 33 delas responderam que não, enquanto sete, afirmaram que sim. $\mathrm{O}$ aborto espontâneo ocorre de forma involuntária, comumente devido a problemas de saúde da mulher ou do feto, sendo a toxoplasmose um possível fator que pode influenciar na ocorrência de abortos principalmente quando a infecção acontece no primeiro trimestre da gestação ${ }^{(19)}$.

No que diz respeito à relação de abortamento em gestação anterior e soropositividade para toxoplasmose, no estudo realizado por Leão et al. ${ }^{(20)}$, de 36 gestantes com história obstétrica de abortamento, 26 tinham sorologia positiva, perfazendo prevalência de $72,2 \%$, contra $70,4 \%$ encontrados no grupo sem tal antecedente.

Com relação à criação de gatos, 10 delas confirmaram que criavam gatos, sabe-se que a toxoplasmose é uma zoonose, ou seja, uma doença transmitida ao homem pelos animais. $O$ gato é o hospedeiro definitivo do protozoário, portanto era de interesse da pesquisa saber quantas gestantes possuíam gatos em casa, e se haviam contato com o felino ${ }^{(21)}$.

Vale reforçar os dados já existentes na literatura os quais demonstram a importância do papel desem- 
Moura DS, Oliveira RCM, Matos-Rocha TJ. Toxoplasmose gestacional: perfil epidemiológico e conhecimentos das gestantes atendidas na unidade básica de saúde de um município alagoano. Arq Med Hosp Fac Cienc Med Santa Casa São Paulo. 2018;63(2):69-76.

penhado pelos felídeos na transmissão e manutenção do ciclo do T. gondii na natureza e através de relatos sobre a ocorrência da toxoplasmose humana e contato com gatos ${ }^{(22)}$.

Em relação aos hábitos de vida das mulheres suscetíveis entrevistadas, foi observado que as práticas de risco relacionadas ao consumo e ao manuseio dos alimentos foram mais comumente relatadas do que as de contato com gatos, salientando que devem ser enfatizadas as mensagens em relação ao consumo de carne e hortaliças cruas, e, particularmente, ao manuseio das carnes cruas ${ }^{(23)}$.

Em um estudo realizado por Camara et al, 2015(24) na cidade do Caxias/Maranhão evidenciou que o consumo de carne crua teve associação significativa com a sororeatividade para T. gondii nessas gestantes, demonstrando que há diferenças significativas entre os grupos, ou seja, houve risco de se contrair toxoplasmose no grupo que comeu carne crua, porém, o risco não pode ser estimado.

Resultado difere do estudo anterior, onde não foi encontrada associação entre a toxoplasmose e os diferentes hábitos de comportamento, como ingestão de carnes cruas ou mal cozidas, ingestão de vegetais crus, ingestão de salames coloniais, manipulação de terra ou areia, presença de horta em casa, presença de gatos em casa ${ }^{(4)}$.

Ao serem perguntadas se a água ingerida é filtrada ou mineral, 09 (23\%) delas responderam que não. Diante de tais informações, conclui-se que é imprescindível que água para consumo humano seja tratada adequadamente, com filtração e desinfecção. Logo, é relevante saber a origem da água consumida pelas gestantes durante a gestação. A água deve ser tratada (filtrada e descontaminada), sujeita a tratamento e a controle de qualidade. No caso de água proveniente de poço artesiano, é essencial que tal água seja fervida e filtrada antes de ser consumida ${ }^{(1)}$.

Deve-se alertar a gestante que a literatura tem registrado surtos de toxoplasmose através da ingestão de água, que acontece porque os felídeos (gatos) eliminam as formas de oocistos que são resistentes permanecendo no solo e assim, podendo eventualmente contaminar a água, esse tipo de contaminação é visto principalmente em poços artesianos e/ou caixas de água ${ }^{(25)}$.

As gestantes foram questionadas quanto ao tipo de leite consumido, já que o leite pode estar relacionado diretamente como fator de infecção pelo $T$. gondii, assim, $16(40 \%)$ gestantes relataram ingerir leite fervido. Nesse caso, percebe-se que algumas gestantes ainda desconhecem a importância de tratar o leite antes de ingeri-lo. Contudo, deve-se propor que a paciente antes de consumir, observe se está devidamente pasteurizado, caso não, colocar em fervura ${ }^{(26)}$.

A presença de taquizoítos T. gondii foi relatada no leite de ovelhas, cabra e vacas. Apesar do leite cru não ser a principal via de transmissão da toxoplasmose, ele pode veicular o parasito durante a fase aguda de parasitemia, portanto deve ser ingerido somente após a pasteurização ou fervura ${ }^{(27)}$.

A toxoplasmose pode ser adquirida pela ingestão de alimentos contaminados, em especial carnes cruas ou mal passadas, principalmente de porco e de carneiro contendo cistos, e vegetais que abriguem os oocistos, por terem tido contato com as fezes eliminadas por felídeos contendo oocistos ou material contaminado por elas mesmas ${ }^{(28-29)}$.

No estudo descrito por Camara et al, 2015 ${ }^{(24)}$ não foi encontrada associação entre a infecção por T. gondii e os diversos hábitos de comportamento, presença de horta em casa, presença de gatos em casa e contato com gatos durante a fase adulta, ingestão de vegetais crus, ingestão de linguiça caseira e leite in natura e manipulação de terra ou areia pelas gestantes.

De 40 gestantes, 34 delas desconhece qualquer meio de prevenção da toxoplasmose e apenas seis conhecem formas de prevenir a doença. Nesse caso, nota-se que a maioria das gestantes não sabe os métodos de prevenção, desta forma, propõe-se que nas consultas de pré-natal, independente de idade gestacional, sejam abordados temas referentes o assunto, buscando assim, um maior conhecimento das mulheres acerca da T. gondii.

Um estudo realizado por Camara et al, $2015^{(24)}$ cabe destacar a susceptibilidade (IgG e IgM não reagentes) de $22,1 \%$ das gestantes em nosso meio, deixando as mulheres suscetíveis ao T. gondii, consequentemente, estão mais expostas a adquirir a primo infecção durante a gravidez, evidenciando a importância de implementar ações de prevenção e controle, visando evitar as infecções congênitas.

Sugere-se que, em um programa de prevenção primária para toxoplasmose, institua-se o aconselhamento de todas as gestantes sobre as medidas para evitar a infecção durante a gravidez. Sabe-se que geralmente as grávidas acatam esse tipo de recomendação quando o esclarecimento é realizado, uma vez que o alvo visado é o bem-estar do concepto. Assim, não apenas recomendações específicas devem ser indicadas pelos profissionais responsáveis pela assistência pré-natal, como também recomendações por escrito deveriam ser distribuídas para todas as gestantes na primeira consulta pré-natal ou preferentemente na consulta pré-concepcional $^{(30)}$.

De qualquer forma, independente do tipo de programa que possa ser adotado, o fundamental é que não podemos estar omissos em relação ao problema da toxoplasmose congênita. Qualquer que seja a estratégia preventiva deve ser incorporada à rotina pré-natal em toda a população ${ }^{(31)}$. 
Moura DS, Oliveira RCM, Matos-Rocha TJ. Toxoplasmose gestacional: perfil epidemiológico e conhecimentos das gestantes atendidas na unidade básica de saúde de um município alagoano. Arq Med Hosp Fac Cienc Med Santa Casa São Paulo. 2018;63(2):69-76.

De acordo com o estudo publicado por Souza et al, $2010^{(32)}$ é de competência do Ministério da Saúde, estabelecer políticas e normas para oferta do pré-natal de boa qualidade, além dos equipamentos e instrumentos para realização de consultas e exames, deve se levar em conta ainda que a capacitação adequada de todas as pessoas que atendem a mulher no seu percurso pela unidade de saúde.

No entanto, Silva e Okasakim (2012) ${ }^{(33)}$, relatam em seu estudo que em locais onde a Estratégia de Saúde da Família está implantada o acompanhamento é realizado pela equipe multiprofissional. As gestantes constituem o foco principal do processo de aprendizagem, não deixando, contudo de serem vistas em seu contexto familiar e social.

\section{Conclusão}

Com base nos resultados apresentados, fica clara a necessidade de implantação de programas de educação em saúde para prevenção da toxoplasmose. Portanto, cabe aos profissionais de saúde aperfeiçoar o acompanhamento pré-natal das gestantes atendidas nessa unidade, executando estratégias de educação em saúde, como realização de palestras sobre doenças congênitas, distribuição de cartilhas e aperfeiçoamento das informações dadas às gestantes durante as consultas de pré-natal. Tal educação, ou prevenção primária, consiste na disseminação do conhecimento sobre a doença e seus meios de prevenção.

\section{Referências}

1. Brasil. Ministério da Saúde. Secretaria de Vigilância em Saúde Departamento de Vigilância Epidemiológica. Doenças infecciosas e parasitárias: guia de bolso. $8^{\mathrm{a}}$ ed. rev. Brasília: Ministério da Saúde; 2010. 444 p. (Série B. Textos Básicos de Saúde)

2. Porto AMF, Amorim MMR, Coelho ICN, Santos LC. Perfil sorológico para toxoplasmose em gestantes atendidas em maternidade. Rev Assoc Med Bras. 2008; 54(3):242-8.

3. Barbaresco AA, Costa TL, Avelar JB, Rodrigues IMX, Amaral $\mathrm{WN}$, Castro AM. Infeccoes de transmissão vertical em material abortivo e sangue com ênfase em Toxoplasmose gondii. Rev Bras Ginecol Obstet. 2014; 36(1):17-22.

4. Bittencourt LHFB, Lopes-Mori FMR, Mitsuka-Breganó R, Valentim-Zabott M, Freire RL, Pinto SB, et al. Soroepidemiologia da toxoplasmose em gestantes a partir da implantação do Programa de Vigilância da Toxoplasmose Adquirida e Congênita em municípios da região oeste do Paraná. Rev Bras Ginecol Obstet. 2012; 34(2):63-8

5. Kurihara CH, Silva CJ. Diagnostic leaf to evaluate the nutritional status of Jatropha. Rev Ceres. 2015; 62(6):607-13.

6. Pessanha TM, Carvalho M, Pone MVS, Gomes Júnior SC. Abordagem diagnóstica e terapêutica da toxoplasmose em gestantes e as repercussões no recém-nascido. Rev Paul Pediatr. 2011; 29(3):341-7.

7. Lopes-Mori FMR, Mitsuka-Breganó R, Capobiango JD, Inoue IT, Reiche EMV, Morimoto HK, et al. Programas de controle da toxoplasmose congênita. Rev Assoc Med Bras. 2011; 57(5):594-9.

8. Mioranza SL, Meireles LR, Mioranza EL, Andrade Júnior HF.
Evidência sorológica da infecção aguda pelo Toxoplasma gondii em gestantes de Cascavel, Paraná. Rev Soc Bras Med Trop. 2008; 41(6):628-34.

9. Andrade GMQ, Vasconcelos-Santos DV, Carellos EVM, Romanelli RMC, Vitor RWA, Carneiro ACAV, et al. Toxoplasmose congênita em filho de mãe cronicamente infectada com reativação de retinocoroidite na gestação. J Pediatr. (Rio J.)2018; 86(1):85-8.

10. Inagaki ADM, Cardoso NP, Lopes RJPL, Alves JAB, Mesquita JRF, Araújo KCGM, et al. Análise espacial da prevalência de toxoplasmose em gestantes de Aracaju, Sergipe, Brasil. Rev Bras Ginecol Obstet. 2014; 36(12):535-40.

11. Amendoeira MRR, Camillo-Coura LF. Uma breve revisão sobre toxoplasmose na gestação. Sci Med. 2010; 20(1):113-9.

12. Avelino MM, Campos Jr D, Parada JCB, Castro AM, Medical School of the Federal University of Goiás, Institute of Tropical Pathology and Public Health of the Federal University of Goiás, et al. Pregnancy as a risk factor for acute toxoplasmosis seroconversion. Eur J Obstet Gynecol Reprod Biol. 2003; 108(1):19-24.

13. Spalding SM, Amendoeira MRR, Ribeiro LC, Silveira C, Garcia AP, Camillo-Coura L. Estudo prospectivo de gestantes e seus bebês com risco de transmissão de toxoplasmose congênita em município do Rio Grande do Sul. Rev Soc Bras Med Trop. 2003; 36(4):483-91.

14. Silva LR, Okazaki ELFJ. Enfermagem e a prevenção da Toxoplasmose durante a gestação. Rev Enferm UNISA. 2012; 13(1):43-7.

15. Silva VLM, Camargo Júnior KR. Em busca do feto saudável: ideias, marcas e coisas na reconstrução do diagnóstico da toxoplasmose. Hist Cienc Saude-Manguinhos. 2013; 20(2):643-51.

16. Liu Q, Wei F, Gao S, Jiang L, Lian H, Yuan B, et al. Toxoplasma gondii infection in pregnant women in China. Trans $\mathrm{R}$ Soc Trop Med Hyg. 2009; 103(2):162-6.

17. Lopes FMR, Mitsuka-Breganó R, Gonçalves DD, Freire RL, Karigyo CJT, Wedy GF, et al. Factors associated with seropositivity for anti-Toxoplasma gondii antibodies in pregnant women of Londrina, Paraná, Brazil. Mem Inst Oswaldo Cruz. 2009; 104(2):378-82.

18. Hung CC, Fan CK, Su KE, Sung FC, Chiou HY, Gil V, et al. Serological screening and toxoplasmosis exposure factors among pregnant women in the Democratic Republic of Sao Tome and Principe. Trans R Soc Trop Med Hyg. 2007 Feb;101(2):134-9.

19. Isabel TF, Costa PI, Simões MJS. Toxoplasmose em gestantes de Araraquara/SP: análise da utilização do teste de avidez de IgG anti-Toxoplasma na rotina do pré-natal. Sci Méd. 2007; 17(2): 57-61.

20. Leão PRD, Meirelles Filho J, Medeiros SF. Toxoplasmose: soroprevalência em puérperas atendidas pelo Sistema Único de Saúde. Rev Bras Ginecol Obstet. 2004; 26(8): 627-32.

21. Galanakis E, Manoura A, Antoniou M, Sifakis S, Korakaki E, Hatzidaki E, et al. Outcome of toxoplasmosis acquired during pregnancy following treatment in both pregnancy and early infancy. Fetal Diagn Ther. 2007; 22(6):444-8.

22. Montoya JG, Remington JS. Management of Toxoplasma gondii infection during Pregnancy. Clin Infect Dis. 2008; 47(4):554-66.

23. Carellos EVM, Andrade GMQ, Aguiar RALP. Avaliação da aplicação do protocolo de triagem pré-natal para toxoplasmose em Belo Horizonte, Minas Gerais, Brasil: estudo transversal em puérperas de duas maternidades. Cad. Saúde Pública. 2008; 24(2):391-401.

24. Camara, JT, Silva MG, Castro AM. Prevalência de toxoplasmose em gestantes atendidas em dois centros de referência em uma cidade do Nordeste, Brasil. Rev Bras Ginecol Obstet. 2015; 37(2):64-70

25. Leal PDS. Toxoplasma gondii Doenças intercorrentes em cães sororreagentes provenientes do Rio de Janeiro, RJ. Tese (Doutorado). Rio de Janeiro: Universidade Federal Rural do Rio de Janeiro, 2014 
26. Lago EG, Carvalho RL, Jungblut R, Silva VB, Fiori RM. Screening for Toxoplasma gondii antibodies in 2,513 consecutive parturient women and evaluation of newborn infants at risk for congenital toxoplasmosis. Sci Med. 2009; 19(1):27-34.

27. Suárez-Hernándes M, González-Fernández A, Gardón-Quirola BY, Martínez-Sánchez R Infección y enfermedad por Toxoplasma gondii en animales y humanos en 23 años de observación en la provincia de Ciego de Ávila, Cuba. Rev Biomed (México). 2005; 16(1): 21-7.

28. Ragozo AM, Yai LE, Oliveira LN, Dias RA, Gonçalves HC, Azevedo SS, et al. Isolation of Toxoplasma gondii from goats from Brazil. J Parasitol. 2009; 95(2): 323-6.

29. Wanderley FS, Porto JW, Câmara DR, da Cruz NL, Feitosa BC, Freire RL, DE Moraes EP, Mota RA. Experimental vaginal infection of goats with semen contaminated with the "CPG" strain of Toxoplasma gondii. J Parasitol. 2013; 99(4): 610-3.
30. Scherer LM, Borenstein MS, Padilha MI. Gestantes/puérperas com hiv/aids: conhecendo os déficits e os fatores que contribuem no engajamento para o autocuidado. Esc Anna Nery Rev Enferm. 2009; 13(2):359-65.

31. Chêne G, Thiébaut R. Options for clinical trials of pre and post-natal treatments for congenital toxoplasmosis. Mem Inst Oswaldo Cruz. 2009; 104(2): 299-304.

32. Souza CO, Tashima NT, Silva MA, Tumitan ARP. Estudo transversal de toxoplasmose em alunas de um curso superior da região de Presidente Prudente, Estado de São Paulo. Rev Soc Bras Med Trop. 2010; 43(1):59-61.

33. Silva LR, Okazaki, ELFJ. Enfermagem e a prevenção da toxoplasmose durante a gestação. Rev Enferm UNISA. 2012; 13(1):43-7.

Trabalho recebido: $31 / 10 / 2017$

Trabalho aprovado: 17/06/2018 